\title{
Perfil de competencias de los docentes de la unidad curricular Morfofisiopatologia del programa medicina de la Universidad Nacional Experimental Francisco de Miranda
}

\section{Proficiency profile of teachers in the curricular unit Morphofysiopathology of the Francisco de Miranda National Experimental University medicine program}

\author{
Reina Rivero Boyer \\ reinaribo@gmail.com \\ Universidad Nacional Experimental Francisco de Miranda \\ Venezuela
}

Recibido: 18 de octubre del 2018

Aprobado: 20 noviembre del 2018

\begin{abstract}
RESÚMEN
El propósito de esta investigación es determinar el perfil de competencias de los docentes de la unidad curricular Morfofisiopatologia del programa medicina UNEFM. La presente investigación en su dimensión epistemológica se acoge el paradigma cualitativo- interpretativo adopta como enfoque metodológico la etnografía. Para la recolección de datos están: La observación participante la entrevista no estructurada profunda. En el análisis se procedió a la clasificación, codificación y categorización de las unidades. En cuanto a la validez y confiablidad, se toma en cuenta las categorías descriptiva. Los resultados evidencian que los docentes actuales manifiestan habilidades psicológicas, pedagógicas y comunicativas. Las estrategias metodológicas son de tipo constructivista, es importante mencionar que no se aprovechan recursos como la plataforma tecnológica, ya que los Docentes y participantes refieren usar poco o no usar la plataforma tecnológica, por lo que se hace necesario implementar estrategias para fomentar el uso por parte de los docentes de este recurso.
\end{abstract}

Descriptores: Perfil docente; unidad curricular; medicina, morfofisiopatología; dimensión epidemiológica. 


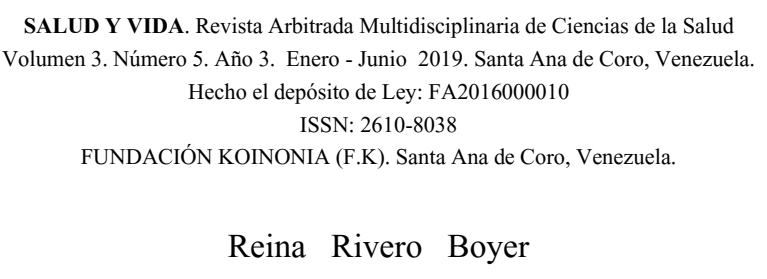

The purpose of this research is to determine the competency profile of teachers in the curricular unit Morphophysiopathology of the UNEFM medicine program. The present investigation in its epistemological dimension welcomes the qualitative-interpretative paradigm adopts ethnography as a methodological approach. For the collection of data are: The participant observation the deep unstructured interview. In the analysis, the classification, coding and categorization of the units was carried out. In terms of validity and reliability, the descriptive categories are taken into account. The results show that current teachers manifest psychological, pedagogical and communicative skills. The methodological strategies are constructivist, it is important to mention that resources such as the technological platform are not used, since teachers and participants refer to using little or not using the technological platform, so it is necessary to implement strategies to encourage use by part of the teachers of this resource.

Descriptors: Teacher profile; curricular unit; medicine, morphophysiopathology; epidemiological dimension.

\section{INTRODUCCION}

Los avances en las nuevas tecnologías, los cambios en los procesos económicos, sociales y políticos. Aunado a esto la globalización influyen en la educación universitaria, que tiene como reto mejorar el desempeño de sus docentes con las nuevas competencias, la cual obliga a repensar el proceso educativo, para uno de liberación y transformación de la realidad de los educandos. De esta manera, surgen las competencias educativas las cuales se basan tanto en la economía como en la administración. Así mismo, intentan aproximar la educación a estas disciplinas en un tiempo, para la creación de mejores destrezas en los estudiantes, para que participen en la actividad productiva. (Argudín, 2014, p. 7). En este sentido, las necesidades y demandas educativas requieren ahora de una atención urgente en forma directa y permanente.

La importancia de los valores y las competencias viene reseñada por múltiples autores e instituciones tal como se recoge en la declaración mundial sobre la educación superior UNESCO $(1998, p, 01)$ en el siglo XXI visión y acción. "En los albores del nuevo siglo se observa una demanda de educación superior, sin precedentes, acompañada de una gran diversificación de la misma, y una mayor toma de conciencia de la importancia fundamental que este tipo de educación reviste para el 


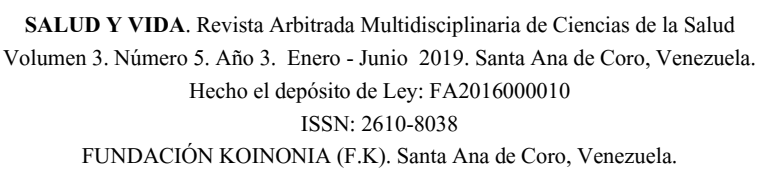

Reina Rivero Boyer

desarrollo socio cultural y económico y para la construcción del futuro de cara al cual, las nuevas generaciones deberán estar preparadas con nuevas competencias nuevos conocimientos e ideales". Es por eso, que el presente estudio permite describir el perfil de competencias de los docentes de la unidad curricular Morfofisiopatologia del programa Medicina de la UNEFM (Universidad Experimental Francisco de Miranda) Modalidad de ADI (Aprendizaje Dialógico Interactivo).

\section{IDEA CENTRAL}

En todo proceso de innovación, cambio reforma educativa el profesorado universitario es uno de los elementos nucleares a considerar, no pudiendo desarrollar una concepción de la educación superior centrada en el logro de las competencias, en el aprendizaje del alumno, en la innovación como medio para alcanzar la calidad y la excelencia. Sin incidir de manera clara en el profesorado y en sus competencias. Así todas estas modificaciones del panorama universitario generan, a su vez, la necesidad de delimitar las competencias que precisa al profesor universitario para desarrollar adecuadamente las funciones. Derivados de este nuevo escenario de actuación profesional planteándose como tarea ineludible revisar la formación necesaria inicial y continua que debe acreditar al profesor universitario en este nuevo contexto a partir del perfil de competencias que se está configurando Mastorell (2011, p.01).

Esta investigación pretende sistematizar las principales innovaciones curriculares desarrolladas en la Universidad Nacional Experimental "Francisco de Miranda", a saber: modalidad mixta, Aprendizaje Dialógico Interactivo, programa del desarrollo integral del personal académico de la UNEFM y la transformación de los currículos basados en perfil por competencia. Es importante mencionar que esta Universidad en Venezuela es reconocida por su trayectoria por su formación de médicos cirujanos con dos modalidades: La primera es la modalidad tradicional, la cual nace al ser creada esta universidad y la modalidad de Aprendizaje Dialógico Interactivo (ADI), es 


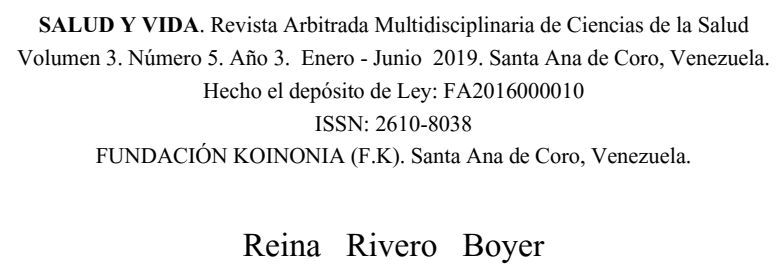

realmente joven la cual ofrece una gama de herramientas y recursos necesarios para la formación de médicos cirujano.

En este sentido, la presente investigación se desarrolla en la UNEFM la cual surge de la observación no estructurada experiencial, donde encontramos docentes en el Programa de Medicina por los cuales surge la inquietud de determinar el perfil de competencias permitiendo observar evaluar el desempeño y la posición del profesor como facilitador en la construcción de competencias. Permitiendo describir de manera pormenorizada el perfil de competencia de los docentes de la unidad utilizando procedimientos de corte cualitativo, los resultados obtenidos nos aportan un perfil de profesor universitario deseable, lo que nos servirá como guía o modelo al momento de la selección de los docentes de la unidad curricular. Ante esta realidad, surge la necesidad de investigar el perfil del docente de la unidad curricular Morfofisiopatologia del Programa de Medicina.

Todo lo cual nos lleva al planteamiento de las siguientes interrogantes, en la presente investigación:

1) ¿Cuál es el perfil de competencias que tiene el docente?

2) ¿Cuáles es el perfil de competencias que utiliza el docente?

3) ¿Cuáles son las estrategias que utiliza el docente en la unidad curricular Morfofisiopatologia?

4) ¿Cuál es la opinión que tienen los estudiantes de las competencias que aplica el docente en la unidad curricular Morfofisiopatologia?

5) ¿Cuáles materiales didácticos utiliza el docente para la aplicación de sus competencias?

\section{Propósitos}

\section{Propósito General}

Describir el Perfil de Competencias de los Docentes de la Unidad Curricular Morfofisiopatologia de Medicina en la UNEFM. Municipio Miranda, Estado Falcón. 


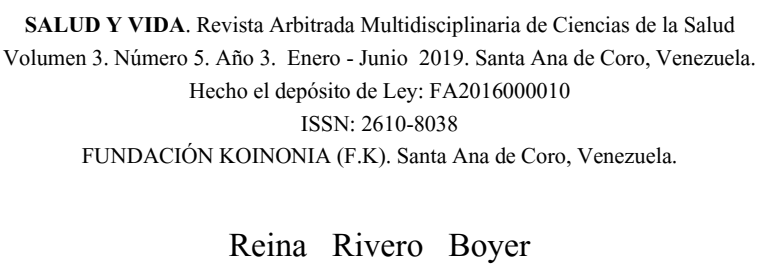

\section{Propósitos Específicos.}

Identificar las competencias profesionales de los docentes en la Unidad Curricular de Morfofisiopatologia de Medicina.

Determinar en su desempeño docente las estrategias metodológicas utilizadas en el desarrollo de sus competencias docente en la Unidad Curricular Morfofisiopatologia de Medicina.

Analizar la opinión que tienen los estudiantes de las competencias que desempeña el docente de la unidad curricular Morfofisiopatologia de Medicina.

\section{Justificación de la Investigación.}

La profunda transformación en el contexto educativo debe producirse a partir del apoyo de las autoridades en las instituciones además de un cambio de actitudes y los planteamientos por parte de los profesores. En este sentido, el sistema educativo debe adaptarse a los cambios sociales y replantear el papel que actualmente debe desempeñar el profesor, las competencias que deben poseer para desenvolverse en la sociedad de la información. Es importante recordar que las competencias parten del marco conceptual que provee la institución con su misión y sello característico .Por su parte, las nuevas formas de enseñanza y de aprendizaje exigen habilidades como la investigación ,búsqueda, estudio, invención, adaptación flexibilidad, creatividad, junto con actitudes de tolerancia a la frustración, que pueden promover los constantes cambios que se prevén para esta era (Yendrey,1999, p. 3).

Por consiguiente, se puede afirmar que la presente investigación cumple con los criterios de relevancia científica por cuanto los resultados pueden contribuir al diseño del perfil profesional deseable del docente de Morfofisiopatologia, determinar si existe la necesidad de crear planes para la formación de dichos docentes, además puede proporcionar criterios para una evaluación y selección más objetiva de los profesores de Morfofisiopatologia y de los que aspiren dictar 
la asignatura. Desde el punto de vista social los resultados pueden contribuir a implementar acciones a la capacitación y mejoramiento académico de los docentes lo que incidirá en un beneficio de la comunidad estudiantil que a su vez requiere una formación acorde a la realidad nacional e internacional cada vez más restringido y competitivo.

Por lo tanto, se justifica la presente investigación desde el punto de vista teórico, ya que facilita conocimientos actualizados sobre el perfil de competencias de los docentes que imparten la unidad curricular como paradigma emergente, dando un aporte para docentes, estudiantes e investigadores en la educación universitaria, con enfoque educativo. De igual forma servirá como punto de partida para otras investigaciones y así aportar conocimientos aquellos campos de estudio de la educación universitaria maestrías de educación universitaria y las distintas instituciones universitarias con sus determinadas modalidades presencial a distancia o semipresencial. Así mismo, sus resultados podrán contribuir para investigaciones posteriores dejando la posibilidad de profundizar, del mismo modo pretende estimular el interés de los participantes y facilitadores en el tema planteado.

En lo metodológico, esta investigación desde el paradigma interpretativo aportara información sobre el perfil docente a partir de la profundización de la realidad de la experiencia de los actores claves de esta investigación. Aspectos importantes, en el estudio de las acciones humanas y de la vida social. Así como la comprensión de lo único y pertinente del docente en su ejercicio profesional ya que la etnografía facilitara a los lectores desde lo antropológico la descripción del ejercicio docente en la unidad curricular de Morfofisiopatologia de igual forma el estudio aporta conocimientos que servirán de base para otras investigaciones que aborden problemas similares.

Por consiguiente, desde el punto de vista práctico, contribuirá a cualquier intención nacional o regional, para la investigación en cuanto al perfil de competencias del docente. La razón de esto, se basa en las transformaciones 
acaecidas actualmente en torno al tema de investigaciones de la educación universitaria a nivel nacional exigen cada día más la actualización, innovación y supervisión, así como el abordaje holístico del contexto social que rodea a los estudiantes, lo anteriormente expuesto concuerda con las nuevas tendencias.

\section{MARCO REFERENCIAL TEÓRICO}

Esta investigación aborda en sus aspectos teóricos, corrientes de pensamientos y estudios relacionados con el perfil de competencias del docente universitario como nuevo enfoque de los paradigmas educativos, dando lugar a importantes desafíos desde una educación tradicional a una emergente. Esta constituye una propuesta que parte del aprendizaje significativo y se orienta a la formación humana integral como condición esencial a todo proyecto. Pues el docente de este siglo está llamado a una misión más comprometida a una concepción del acto educativo de forma holística, liberadora, critica y reflexiva para la transformación de la realidad.

En relación a este estudio, existen investigaciones acerca de la descripción del Perfil de Competencias específicamente en un grupo de docentes de la Universidad del Zulia, el cual trata del desempeño de la función de investigador, determinando la relación de dicho perfil con los índices de producción intelectual. La población seleccionada fue los investigadores adscritos al centro de Documentación e Investigación Pedagógica, a quién se aplicó una ficha de datos bibliográficos y un cuestionario tipo escala, sometido al juicio de expertos y a la prueba de confiabilidad, concluyendo que los investigadores de la muestra poseen un alto nivel de competencias genéricas y técnicas. Pírela y otros (2006, p. 8).

\section{Perfil profesional}

El perfil profesional (Guedez, 1980) lo define, como el conjunto de características pretendidas por el empleador, las cuales deben reflejar las exigencias del mercado ocupacional, en termino de los requisitos que definan las habilidades, 
las destrezas, los rasgos de personalidad, la conformación física y el nivel de educación inherentes al desempeño profesional. (p 17).Para (Díaz, y otros 1999) el perfil profesional lo componen tanto conocimientos, habilidades y actitudes ( $p .87)$. Considera al perfil como imagen y modelo de logro en una profesión de allí la importancia de la totalidad de conocimientos y habilidades necesarias para un profesional de cualquier carrera de quien se espera maestría en su ejercicio laboral. (Fernández, 2004, p. 121).

Por otra parte el perfil del docente universitario en el siglo XXI, comprende entre otras competencias: El dominio de conocimientos de su disciplina, para poder orientar el proceso de aprendizaje, evaluar formativamente, incentivar y motivar a los participantes; saber hacer, a fin de que al actuar y reflexionar en sobre la acción, pueda construir su propio pensamiento profesional, asumirlo y actuar en consecuencia ; innovador en su praxis docente, lo que implica reflexionar e investigar, integrando el conocimiento interdisciplinario y pedagógico el camino para la mejora continua, dominar las herramientas relacionadas con el currículo (diseño, planificación y gestión del mismo). Además de favorecer entre los participantes un clima de motivación hacia un aprendizaje de calidad (Palomares, 2007:144).

De igual manera, este docente ha de dominar otros idiomas nuevas tecnologías y estar dispuesto a la movilidad y a la realización de intercambios, a fin de integrarse a un nuevo modelo docente; manejar estrategias metodológicas y comunicativas; ser consecuente con sus ideas planteamientos y sentimientos analizando la realidad de forma reflexiva y critica, al mismo tiempo que proporciona alternativas ante los diversos problemas que se presentan en el ejercicio docente. A su vez promoverá actitudes positivas hacía la diversidad, la apertura y el respeto hacia las diferencias individuales Por tal motivo, el docente ha de trabajar en conjunto con sus colegas y fortalecer el aprendizaje colaborativo entre sus participantes, tener habilidad comunicativa y de relación necesarias en 
SALUD Y VIDA. Revista Arbitrada Multidisciplinaria de Ciencias de la Salud

Volumen 3. Número 5. Año 3. Enero - Junio 2019. Santa Ana de Coro, Venezuela.

Hecho el depósito de Ley: FA2016000010

ISSN: 2610-8038

FUNDACIÓN KOINONIA (F.K). Santa Ana de Coro, Venezuela.

Reina Rivero Boyer

el ejercicio docente y estar comprometidos con la dimensión ética de su profesión . Benito (2005, p. 19).

Por otro lado, el docente es un organizador y mediador en el encuentro del estudiante con el conocimiento. Este docente tiene competencias de conocimientos teóricos pertinentes acerca del aprendizaje de desarrollo y de comportamiento humano; posee y promueve valores morales y éticos, actitudes que fomentan el aprendizaje y buenas relaciones humanas; dominio de los contenidos o de las materias que enseña. Así como, manejo de estrategias mediadoras; conocimiento sobre el aprendizaje de las ciencias; capacidad crítica y fundamentada de su enseñanza habitual; planifica ,aplica instrumentos de evaluación de coevaluación, autoevaluación y evaluación externa. Además de realizar investigaciones e innovaciones disciplinarias y psicopedagógicas es facilitador estratégicamente de contenidos y habilidades de dominio; adquiere conocimientos teóricos -prácticos sobre la enseñanza de la materia Díaz y otros (2006, p.3-9)

Por último, el docente inmerso en las transformaciones educativas presentes se perfila como docente reflexivo, flexible, humanista, con manejo de nuevos conocimientos en torno a la evolución, de los contenidos de las unidades curriculares que están a su cargo para garantizar un ejercicio docente de calidad orientar y guiar de manera constructivista sus competencias como docente e irse actualizando de acuerdo a los nuevos paradigmas educativos

\section{Perfil del docente universitario}

Como su denominación lo indica, el nivel de educación universitaria requiere un docente preparado para atender las exigencias y demandas particulares de la institución universitaria. En este sentido, Salcedo (2000, p.9) lo define como el conjunto organizado y coherente de atributos 0 características altamente deseables en un docente universitario, las cuales se expresarían en los conocimientos, las destrezas, actitudes y los valores que le permitan desempeñarse eficientemente, con un sentido creador y crítico, en las funciones 
de docencia, investigación, creación, académica, concebidas estas como unas funciones interdependientes, comprometidas con el logro de la misión de la universidad. Este perfil debe considerar la formación académica, En Venezuela la Constitución de la República Bolivariana de Venezuela establece en su artículo № 104, La educación estará a cargo de personas de reconocida moralidad y de comprobada idoneidad académica. El Estado estimulara su actualización permanente y les garantizara la estabilidad en el ejercicio de la carrera docente, bien sea pública o privada, atendiendo a esta constitución y a la ley.

\section{Competencia}

La palabra competencia se deriva del griego agon, y agonistes, que indica aquel que se ha preparado para ganar en las competencias olímpicas, con la obligación de salir victorioso y por tanto de aparecer en la historia. El areté suprema que anhelaba todo ciudadano griego, era ser triunfador en el combate, adquirir la posición de héroe $y$, por tanto, ver su nombre distinguido en la historia y su imagen recordada en mármol. En principio la educación griega estaba dirigida para alcanzar ese arete la virtud suprema. A partir de Pitágoras y con Platón y Aristóteles, este areté cambia en sentido ser el mejor en el saber el constructor de teorías rectoras de proyectos políticos; las competencias se desplazan desde habilidades y destrezas atléticas para triunfar, hacia exigencias culturales y cognoscitivas. Por lo tanto, en la actualidad, solo las colectividades que han creado las condiciones para producir saberes científicos y tecnológicos son los que hacen protagonismo. Argudín (2005, p.7).

Por otra parte las competencias se vienen abordando en la educación y en el mundo organizacional desde diferentes enfoques, como por ejemplo el conductismo, funcionalismo, el constructivismo y el sistémico complejo. La formación basada en competencias se está convirtiendo en una política educativa internacional de amplio alcance contribuciones conceptuales y metodológicas a las competencias por parte de investigadores de diferentes países desde la época de los años 70 
por ejemplo (chansky 1970:4) el concepto está presente en las políticas educativas de varias entidades internacionales tales como la UNESCO se ha propuesto como una política para la educación superior desde el congreso mundial de educación superior procesos educativos de países como chile ,México actualmente hay en marcha diversos proyectos internacionales como proyecto Tuning (Tobon, 2017, p.17).

Por otra parte algunos autores como Quellet (2000) define las competencias "como el principio de organización de la formación ,la competencia puede apreciarse en el conjunto de actitudes de conocimientos y de habilidades específicas que hacen a una persona capaz de llevar a cabo un trabajo o de resolver un problema particular". (p.37). Para Bogoya (2000) "las competencias son una actuación idónea que emerge en una tarea concreta en un contexto con sentido donde hay un conocimiento asimilado con propiedad y el cual actúa para ser aplicado en una situación determinada, de manera suficientemente flexible como para proporcionar soluciones variadas y pertinentes"(p.11). Según Levy (2000) "Son repertorio de comportamientos que algunas personas dominan mejor que otros los que los hace eficaces en una situación determinada". (p.10).

Conceptos más actualizados definen las competencias de la siguiente manera en este caso para Zabalza (2003,p.70), es el conjunto de conocimientos y habilidades que los sujetos necesitamos para desarrollar algún tipo de actividad según Álvarez (2006, p.23) es el conjunto de conocimientos habilidades y actitudes necesarios para desempeñar una ocupación dada y la capacidad de movilizar y aplicar estos recursos en un entorno determinado para producir un resultado definido.

\section{Clasificación de las competencias}

Según Tobón (2007) una de las clasificaciones más extendidas consiste en dividirlas en competencias en básicas ,genéricas y específicas. Las básicas son las competencias fundamentales para vivir en sociedad y desenvolverse en cualquier 


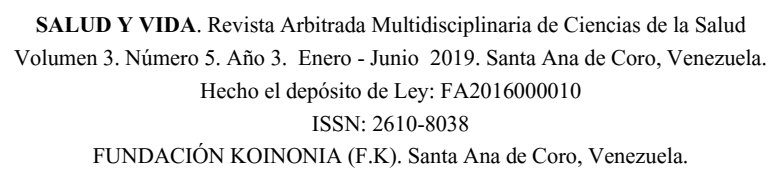

Reina Rivero Boyer

ámbito laboral .Las genéricas son aquellas comunes a varias ocupaciones 0 profesiones por ejemplo la administración de empresas .Las especificas son aquellas competencias propias a una determinada ocupación o profesión. Tienen un alto grado de especialización, así como procesos educativos específicos, tales competencias difieren a las competencias que debería poseer un medico idóneo tales como el chequeo de los signos vitales el diagnostico de salud enfermedad y la implementación de tratamientos pertinentes a diversas enfermedades.(p. 36-45).

\section{Antecedentes históricos del Programa de Medicina}

Con motivo de celebrarse el 26 de julio de 1977, los 450 años de haber sido fundada la ciudad de coro el ejecutivo nacional decide ofrendar a sus habitantes el mejor regalo y mediante decreto publicado en Gaceta oficial de la Republica del 28-01-77 se crea la UNEFM, con sede en coro, capital del estado, dando así a la comunidad la institución que se constituirá en rectora de los quehaceres científicos, tecnológicos, culturales y sociales las primeras autoridades por resolución 282 del ministerio fueron el Doctor Tulio Arens vicerrector académico Pedro Borregales vicerrector administrativo. Inicios académicos 6 meses después, el 23 de abril de 1979 se da inicio al curso de nivelación, llamado posteriormente curso de iniciación universitario la primera carrera en iniciar sus actividades fue Medicina en fecha 15 de octubre de 1973 con 135 alumnos.

\section{Perfil del Docente universitario de la unidad curricular Morfofisiopatologia}

En el caso de la Unidad Curricular Morfofisiopatologia es requisito indispensable ser de profesión Médico Cirujano, y de ser posible con post grado en Universitario Anatomía Patológica. No haber reprobado la unidad curricular Morfofisiopatologia este perfil considera la formación académica. Por otra parte según lo establecido en la Constitución de la República Bolivariana de Venezuela establece en su artículo № 104, La educación estará a cargo de personas de reconocida moralidad y de 


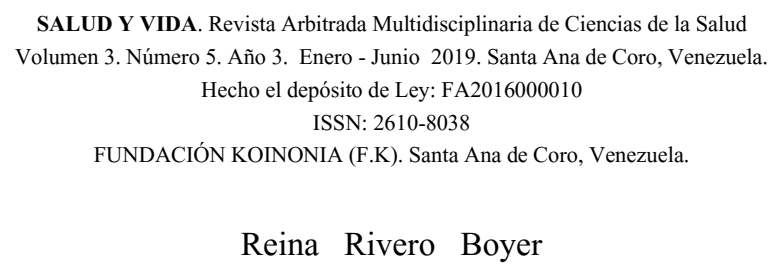

comprobada idoneidad académica. El Estado estimulara su actualización permanente y les garantizara la estabilidad en el ejercicio de la carrera docente, bien sea pública o privada, atendiendo a esta constitución y a la ley.

\section{Descripción general de la asignatura Morfofisiopatología}

El Termino Morfofisiopatologia en el diseño curricular de la carrera de medicina, es realmente nuevo. Este es un programa de la Universidad Nacional Experimental "Francisco de Miranda", cuyo contenido académico ha sido elaborado con un enfoque netamente integracionista y trata de ir a la par con los avances que se operan en las disciplinas científicas interrelacionadas con la medicina. Obedeciendo a este criterio, se considera que el estudio de la enfermedad no debe iniciarse con la anatomía patológica aislada que tiene como base la autopsia y el estudio microscópico de las alteraciones morfológicas que acompañan a los diferentes padecimientos, sino que debe implementarse el estudio de lo patológico con un programa que correlacione las alteraciones morfológicas con los hechos bioquímicos, fisiológicos, epidemiológicos, psicológicos y sociológicos que acompañan a toda enfermedad.

Siguiendo este orden de ideas, esta materia adquiere las características de una disciplina dinámica que desde ahora prepara al futuro médico para relacionar incluso los hechos morfofuncionales con los aspectos clínicos de determinada enfermedad. Basándose en este principio, se plantea el esquema que correlaciona la Morfofisiopatologia con las demás materias afines Morfofisiopatologia I se dicta en el IV semestre consta de cuatro(4) unidades la unidad I introducción al estudio de la enfermedad ,la unidad II alteraciones de las células o tejidos, III Alteraciones genéticas e inmunológicas, IV alteraciones hemodinámicas consta de 12 temas ,Morfofisiopatologia II se dicta en el V semestre consta de 3 unidades la unidad I del sistema cardiovascular, unidad II del sistema digestivo, y la unidad III del sistema respiratorio consta de 30 temas. Morfofisiopatologia III comprende tres (3) unidades de estudio la unidad I neurológico, II Hematopoyético, III Endocrinología y contiene 34 temas serán evaluados de acuerdo a las normativas correspondientes. 


\section{Currículo basado en competencias}

El currículo es el proyecto formativo que se pretende llevar a cabo en una institución educativa, es obtener mejoras en la formación de las personas que participen en él. Cualquier programa universitario ha de servir para mejorar a las personas en todo el amplio espectro de dimensiones en que los estudiantes universitarios puedan mejorar como personas, como futuros profesionales, no se trata pues de aprender cosas sino de formarse, esa es la gran misión de la universidad. El currículo integrado decimos integrado, en el sentido que los proyectos curriculares precisan unidad y coherencia interna. No es un amontonamiento de conocimientos y experiencias sino un proceso caracterizado por una adecuada estructura interna y una continuidad que sea capaz de promover el máximo desarrollo personal y profesional de los estudiantes. Zabalza (2007, p.21).

\section{METODOLOGIA}

En esta metodología se presenta el argumento operante de la investigación a través del cual se establecen los lineamientos que se llevaron a para obtener la información necesaria y dar respuesta a las interrogantes. El paradigma en la presente investigación es de tipo cualitativo y su dimensión es de tipo interpretativo. Esta investigación trata de descubrir, interpretar el perfil de competencias de los Docentes de la Unidad Curricular Morfofisiopatologia de Medicina de la modalidad ADI en la UNEFM.

\section{Paradigma que sustenta la investigación}

Esta investigación se sitúa en el paradigma interpretativo, en la perspectiva antropológica, pues la realidad que se investiga precisa de un estudio descriptivo e interpretativo, por su misma naturaleza y por el propósito planteado. Se acoge a este paradigma interpretativo porque pretende describir el perfil de competencias de los docentes de la unidad curricular Morfofisiopatologia de la UNEFM, a partir de una 
profundización en la experiencia de sus actores, esto resulta necesario en la educación universitaria. Pues, el paradigma interpretativo se centra en el estudio de los significados de las acciones humanas y de la vida social.

\section{Diseño de investigación}

Este estudio es de tipo Etnográfico, rama de la antropología que estudia descriptivamente las culturas. Etimológicamente significa la descripción( grafé ) del estilo de vida de un grupo de personas habituadas a vivir juntas(ethnos).Por tanto el ethnos, que sería la unidad de análisis para el investigador, no solo podría ser una nación, un grupo lingüístico, una región o una comunidad sino cualquier grupo humano que constituya una entidad cuyas relaciones estén reguladas por la costumbre o por ciertos derechos y obligaciones recíprocos. Así, en la sociedad moderna, una familia, una institución educativa, una fábrica, una empresa, un hospital, una cárcel, un gremio obrero, un club social y hasta un aula de clase, son unidades sociales que pueden ser estudiadas etnográficamente.(Martínez, 1998, p.29).

El enfoque etnográfico se apoya en la convicción de que las tradiciones, roles, valores y normas del ambiente en que se vive se van internalizando poco a poco y generan regularidades que pueden explicar la conducta individual y de grupo en forma adecuada, en efecto, los miembros de un grupo étnico, cultural o situacional comparten una estructura lógica 0 de razonamiento que por lo general, no es explicita, pero que se manifiesta en diferentes aspectos de vida. El objetivo inmediato de un estudio etnográfico es crear una imagen realista y fiel del grupo estudiado, pero su intención y mira más lejana es contribuir en la comprensión de sectores o grupos poblacionales más amplios que tienen características similares.(Martínez 1998, p.30). 


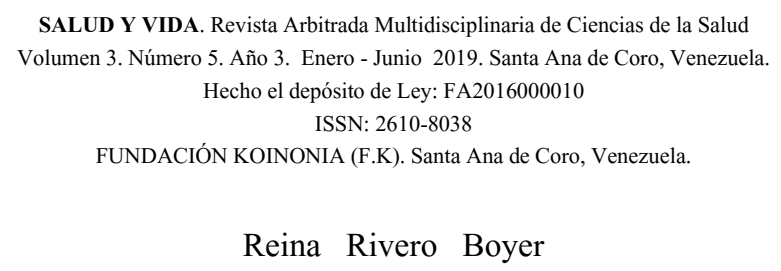

\section{Informantes claves}

Los informantes claves del estudio son 3 Docentes del Programa Medicina de la unidad curricular Morfofisiopatologia de profesión medico ,3 estudiantes de la unidad curricular Morfofisiopatologia. Los docentes son médicos en su mayoría egresados de la misma casa de estudio, los estudiantes son estudiantes regulares de la materia. En cuanto al investigador es egresado a nivel de pregrado como médico General, actualmente Docente y Coordinador de la Unidad Curricular Morfofisiopatologia II.

\section{Espacio geográfico del contexto}

Es en la Ciudad Santa Ana de Coro Estado Falcón modalidad ADI, Anexo a hospital, Edificio Santa Ana, Quinta Santa Eduvigis. Programa Medicina Unidad Curricular Morfofisiopatologia I, II, III.

\section{Tiempo de observación}

Esta comprendido diciembre 2015 a octubre 2017.Correspondiente al periodo académico II 2015 al I 2017.

\section{Técnicas para la recolección de la información}

En la investigación Etnográfica, la información que se busca es aquella que más relación tenga y ayude a descubrir mejor las estructuras significativas que dan razón de la conducta de los sujetos en estudio. El Etnógrafo utiliza, como técnica primaria para recoger la información, las anotaciones de campo tomadas in situ, o después del evento observado, tan pronto como le sea lógico y éticamente posible. Sin embargo, usa un amplio conjunto de técnicas para complementar y corroborar sus notas de campo grabaciones de audio, de video y fotografías. A continuación se mencionan cada una de ellas para la realización de la investigación son las siguientes: 


\section{Observación participante}

En el campo investigativo, la observación participante se entiende como un proceso deliberado, sistemático, dirigido a obtener información en forma directa del contexto donde tiene lugar las acciones (Rojas 2010, p.73).Esta es la técnica clásica primaria y más usada por los etnógrafos para adquirir información. Para ello, el investigador vive lo más que puede con las personas o grupos que desea investigar, compartiendo sus usos, costumbres, estilo y modalidades de vida.(Martinez:1998:63)Para lograr esto, el investigador debe ser aceptado por esas personas, y solo lo será en la medida en que sea percibido como" una buena persona", franca, honesta, inofensiva y digna de confianza.

Al participar en sus actividades corrientes y cotidianas, va tomando notas de campo pormenorizadas en el lugar de los hechos o tan pronto como le sea posible. Posteriormente estas notas se revisan periódicamente con el fin de completarlas(en caso de que no lo estén) y también para reorientar la observación e investigación. El etnógrafo al sumergirse en una observación participativa debe de tratar de responder las preguntas quien, que, dónde, cuándo y por qué alguien hizo algo es decir se consideran importantes los detalles.(Martínez, 1998,p.64).El método etnográfico es utilizado en esta investigación porque nos permite en gran medida interpretar y conocer lo que es el contexto, el proceso enseñanza aprendizaje ver como esos actores sociales protagónicos en este caso docente desarrollan sus prácticas. Nos da la oportunidad para obtener conocimientos que contribuyan a la comprensión el ámbito donde el docente actúa.

\section{Entrevista no estructurada a profundidad}

En esta modalidad no se dispone de un instrumento de preguntas elaboradas previamente. Sin embargo, la investigadora se orienta por unos objetivos preestablecidos, lo que permite definir el tema de la entrevista. Es por eso, que la entrevistadora debe poseer una gran habilidad para formular las interrogantes sin perder la coherencia. (Arias, 2006, p. 73- 74). En esta investigación se utilizó la 
entrevista no estructurada profunda con preguntas abiertas, flexibles, dinámicas y más .De un modo general, una entrevista no estructurada profunda, es aquella en que existe un margen más o menos grande de libertad para formular las preguntas y respuesta. El investigador, no se guía por un cuestionario o instrumento rígido, sino que fluyen con cierto grado de espontaneidad, permitiendo un diálogo más profundo y rico de presentar los hechos en toda su complejidad, captando no sólo las respuestas de lo tratado sobre la investigación, sino también las actitudes, valores y formas de pensar de los entrevistados, a veces inaccesibles por otras vías. Sabino (2007, p. 109).

\section{Diario de campo}

Es uno de los instrumentos que día a día nos permite sistematizar nuestras prácticas investigativas; además nos permite mejorarlas, enriquecerlas y transformarlas. Según (Bonilla y otros, 1997, p.129) el diario de Campo debe permitirle al investigador un monitoreo permanente del proceso de Observación. Puede ser útil al investigador en él toma nota de aspectos que considere importantes para organizar, analizar e interpretar la información que está recogiendo permite enriquecer la relación teoría práctica.

\section{Confiabilidad y validez.}

\section{Confiabilidad}

El concepto tradicional de confiabilidad implica que un estudio se puede repetir con el mismo método sin alterar los resultados, es decir, es una medida de la replicabilidad de los resultados de la investigación. En las ciencias humanas es prácticamente imposible reproducir las condiciones exactas en que un comportamiento y su estudio se llevaron a cabo .Ya Heráclito dijo en su tiempo que nadie se baña dos veces en el mismo rio.(Martínez,2008, p.119). La confiabilidad tiene dos caras, una externa y otra interna: hay confiabilidad externa cuando investigadores, independientes, al estudiar una realidad en tiempos o situaciones diferentes, llegan a los mismos resultados Hay 


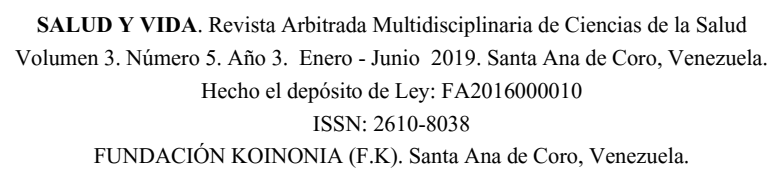

Reina Rivero Boyer

confiabilidad interna cuando varios observadores, al estudiar la misma realidad, concuerdan con sus conclusiones.

Para alcanzar un buen nivel de confiabilidad externa, Goetz y LeCompte (1984) aconsejan:

a) Precisar el nivel de participación y la posición asumida por el investigador en el grupo estudiado.

b) Identificar claramente a los informantes. Estos pueden representar grupos definidos y dar información parcial o prejuiciada.

c) Describir detalladamente el contexto en que se recaban los datos: Contexto físico, social e interpersonal.

d) Identificar los supuestos y metateorias que subyacen en la elección de la terminología y los métodos de análisis para que sea posible una cierta réplica de la investigación.

e) Precisar los métodos de recolección de la información y de su análisis, de tal manera que otros investigadores puedan servirse del informe original como de un manual de operación para repetir el estudio

\section{Validez}

Esta se define por el grado o nivel en que los resultados de la investigación reflejan una imagen clara y representativa de una realidad o situación dada; denominándose así la validez interna. La validez externa consiste en averiguar hasta qué punto las conclusiones de un estudio son aplicables a grupos similares (Martínez, 2008a:119). Es así como, el nivel de validez se aprecia en este estudio de acuerdo al nivel de la habilidad en que se producen las relaciones humanas, con alto sentido de empatía y vinculación. Además, se tendrá presente que a menudo las conclusiones de este estudio, sobre cómo se desarrolla las actividades para determinar el perfil de competencias en este grupo de profesionales, no son comparables con las de otros, pues son específicas y propias del docente en esa situación.

Según Guba y Lincolh (1982) La validez interna, se asegura a través de: 
Permanencia prolongada en el contexto. La permanencia en el escenario por un periodo suficientemente largo permite apropiarse de la cultura de la realidad estudiada, detectar distorsiones introducidas por los participantes o por el mismo investigador, ganar la confianza de las personas. El peligro para el investigador, en estos casos, es" volverse nativo", lo cual lo llevaría a perder la perspectiva de la situación.

Separación periódica de la situación. La separación temporal del investigador durante el desarrollo de la investigación le permite tomar perspectiva y evita el peligro de su conversión en nativo(Santos, 1990). Es oportuno ocupar estos espacios temporales en la redacción de informes, análisis de los resultados u otras actividades que coadyuven a la reflexión.

\section{Criterios de evaluación de la teoría formulada}

Son algunos criterios que se presentan a juicio de la presente investigación se han revelado muy útiles en la validación y evaluación de las teorías. Según Martínez (2008: 105-106), estos son los siguientes:

1. Coherencia interna: Indica que todos los elementos y partes constituyentes de una teoría, se relacionan entre sí sin contradicciones, formando un todo coherente y bien integrado.

2. Consistencia externa: Es la compatibilidad que hay entre la doctrina que constituye la teoría y el conocimiento ya establecido en el mismo campo o campos adyacentes o afines.

3. Comprehensión: Una teoría será mejor que otra si abarca o se relaciona con un amplio campo de conocimientos; es decir, si logra integrar y unificar un vasto espectro de ideas en el área.

4. Capacidad predictiva: La capacidad para hacer predicciones sobre lo que sucederá o no, si se dan ciertas condiciones específicas en ella, aunque la confirmación o contrastación de estas predicciones pueda resultar en extremo difícil, debido de esa teoría. 


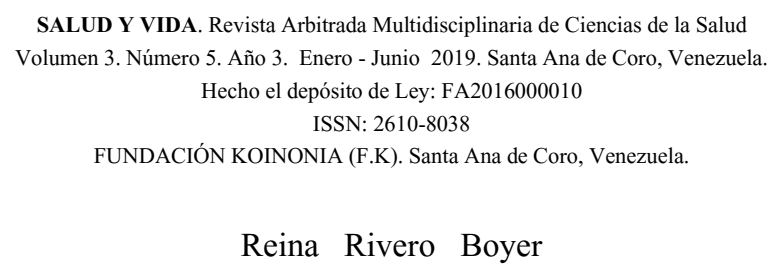

5. Precisión conceptual y lingüística: Debe haber una unidad conceptual; es decir, el universo del discurso debe estar definido y sus predicados deben ser semánticamente homogéneos y conexos.

6. Originalidad: Exige pensar de un modo novedoso, a formular nuevos problemas y a buscar nuevas clases de relaciones y de conocimiento.

7. Simplicidad y parsimonia: En igualdad de condiciones, es preferible la teoría más simple, por su claridad.

8. Potencia heurística: Esta siguiere guiar y generar nuevas investigaciones, planteando nuevos problemas interesantes y facilitando el diseño de estudios y experimentos de gran proyección en el área.

9. Aplicación práctica: Una teoría fácil de aplicar será mejor que otra que, en igualdad de condiciones, es de difícil aplicación.

10. Contrastabilidad: Los postulados, axiomas y derivados sean susceptibles de contrastación, es decir, de un examen, crítica y control que lleven o permitan confirmarla o refutarla. Todo esto depende mucho de la naturaleza del objeto a que se refiere esa teoría.

11. Expresión estética: En la física, por ejemplo, está resultando un lugar común el pensar que la "belleza" de una teoría es a menudo una pista importante hacia su verdad que su correspondencia con los hechos, los cuales pueden constituir una dificultad temporal.

\section{CATEGORIZACIÓN, ANÁLISIS E INTERPRETACIÓN DE LOS RESULTADOS}

En este punto se presentan todos los aspectos a través de los cuales la investigación realizada ha permitido recoger los datos y construir la realidad mediante la información obtenida en el sitio de los acontecimientos, con el objetivo de recoger los datos de una manera naturalista, permitiendo obtener una comprensión adecuada del contexto, presentar los resultados y realizar un análisis cualitativo de la situación planteada. 


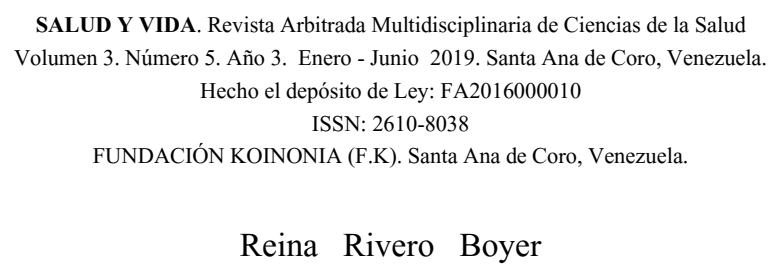

\section{Presentación de los resultados recolectados}

\section{Categorización}

La información obtenida en la presente investigación mediante la observación participante y las entrevistas; fueron organizadas en categorías, así como también sub categorías a continuación se presenta.

\section{Perfil de competencias docentes.}

\section{Sub-categorías:}

-Programa de Desarrollo Integral del Personal Académico (PRODINPA)

-Conocimiento.

\section{Estrategias metodológicas.}

\section{Sub-categorías:}

-Presentación de Seminarios-Clases magistral, Dinámica grupal

-Uso de la plataforma tecnológica.

\section{Opinión de los estudiantes.}

\section{Sub-categorías:}

-Buen docente-Docente critica.

\section{Materiales Utilizados}

-Video beam, pizarra acrílica.

Luego de transcribir, categorizar, codificadar y analizar de forma organizada se estructuro la información en categorías y sub-categorías y temas, se procedió a la contrastación y conjugación de las perspectivas de los temas indicados en esta investigación. 


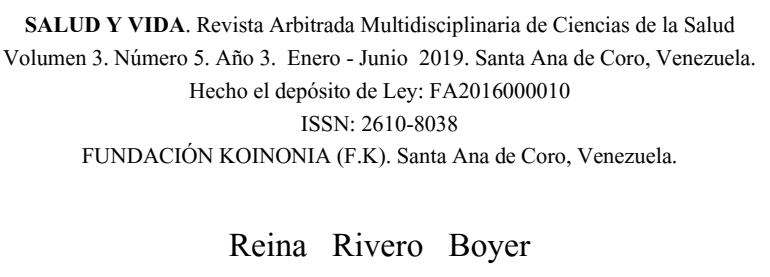

\section{Perfil de competencias docentes}

En cuanto al perfil de competencias docentes expresan que aparte de ser médicos tienen una especialidad. Además manifiestan estar realizando (PRODINPA en la misma institución donde les dan muchas habilidades en lo que tiene que ver con la docencia y la pedagogía como tal una de las cosas es tener el diseño curricular con los objetivos generales de la cátedra y los objetivos específicos para cada tema, suelen organizar el semestre y desde el primer día de clase se le da la normativa a los alumnos con la manera de evaluación los porcentajes las herramientas que es la literatura los recursos y los recursos utilizados video beam.

Otros grupos de participantes en este caso los estudiantes señalan que son buenos docentes, en el desarrollo de las actividades en el aula en este caso los seminarios son críticos, además de ser facilitadores de la información dan orientación de la bibliografía que en otras oportunidades algunos docentes no la dan 0 no les dicen de donde estudiar. Es decir los docentes muestran habilidades tanto por lo expresado por los alumnos como lo observado.

\section{Programa de Desarrollo Integral del Personal Académico (PRODINPA)}

Según lo expresado varios de los docentes señalan estar realizando actualmente el (PRODINPA). Donde adquieren habilidades desde el punto de vista pedagógico lo que les permite mejorar su desempeño, reciben orientación en cuanto al manejo de los contenidos así como también adquirir conocimientos sobre el manejo de las herramientas tecnológicas ,es decir están en proceso de formación docente otros mencionan que aun cuando cumplen funciones docentes no están realizando (PRODINPA), pero si tienen el interés de realizarlo o algún componente docente (Diplomado o maestría) 


\section{Conocimiento}

En otro orden de ideas, los docentes abordados en el estudio señalan tener conocimiento de la materia, bien sea porque los contenidos los lograron ver y aprobar en el desarrollo de su carrera de medicina a nivel de pregrado o los reforzaron en algún postrado o estudio realizado posteriormente, en este caso en su mayoría los docentes abordados señalaron que realizaron estudios de postgrado en medicina familiar en la misma lograr ver contenidos vinculados con la materia.

\section{Estrategias Metodológicas}

-Presentación de Seminarios-Clases magistral, Dinámica grupal

Por otra parte, los estudiantes manifestaron que la estrategia metodológica más utilizada son los seminarios, ellos comentaron recibir apoyo y orientación a la hora de realizarlos. Por lo general, la docente interviene, explica o puede preguntar 0 reforzar o dar de nuevo la clase. Los estudiantes expresan recibir orientación al momento de la preparación de los mismos, es decir que textos consultar para elaborar los contenidos de los seminarios presentados. Los docentes mencionan que la estrategia metodológica más utilizada son los seminarios también utilizan las clases magistrales o las dinámicas grupales, de igual forma algunos docentes expresan tener grupos de estudiantes numerosos.

-Uso de la plataforma tecnológica.

En cuanto al uso de la plataforma tecnológica, los docentes expresan que usan muy poco o no la utilizan la plataforma tecnológica por factor tiempo. Algunos estudiantes expresan que la mayoría de las actividades son presenciales y señalan que no utilizan la plataforma tecnológica sin embargo manifiestan el uso de textos en digital por contar con el recurso de la tablet.

-Opinión de los estudiantes.

En cuanto a la opinión de los estudiantes de los docentes estos expresan que son buenos docentes, en el desarrollo de las actividades docentes son críticos 
en los seminarios, son facilitadores de la información los comparan con otros docentes y refieren que algunos docentes en otros semestres no facilitan nombres o bibliografías a consultar, en el caso de los docentes de Morfofisiopatologia si facilitan la información.

En cuanto a los materiales utilizados, la mayoría de los docentes expresan que en sus presentaciones cuando las realizan y los estudiantes en sus seminarios plantean el uso del video beem.

\section{TEORIZACIÓN}

Con base a la realidad obtenida a partir de la información obtenida de los actores y de la experiencia del investigador, muestra el significado que le da en la investigadora y las teorías establecidas en el marco referencial, con el fin de alcanzar el objetivo interpretativo de este estudio.

En cuanto a las competencias los docentes poseen habilidades pedagógicas, se ve el acompañamiento docente donde este interactúa con el estudiante fomentando y contribuyendo en el proceso de aprendizaje en el aula de clase, sin embargo se requiere ejecutar más acciones para fomentar la innovación en los docentes para mejorar la calidad educativa .

Respecto a las estrategias metodológicas, las teorías que evidencian la investigación en el aula es de tipo constructivista, es decir, una educación basada en nuevos paradigmas con enfoque constructivista, sin embargo es importante mencionar que no se aprovechan recursos de una manera adecuada la plataforma tecnológica ya que los participantes refieren, usar poco o no usar la plataforma tecnológica por lo que se hace necesario implementar estrategias para fomentar el uso por parte de los docentes de este recurso.

Por su parte, los estudiantes opinan que los docentes son críticos, facilitadores de la información y buenos docentes. Es importante que el profesor posea competencias en relación con la preparación susceptibles a generar aprendizaje, lo cual se incrementa cuando el profesor pretende organizar el aprendizaje 
como una construcción de competencias por parte de los estudiantes, se trata de diseñar el desarrollo de los temas con base en actividades a realizar por los estudiantes.

\section{REFERENCIAS CONSULTADAS}

1. Asamblea Nacional de la República Bolivariana de Venezuela.(2009).Ley orgánica de educación.Distribuidor las Piedras .Caracas Venezuela.

2. Asamblea Nacional de la República Bolivariana de Venezuela,2009 Constitución de la República Bolivariana de Venezuela. Ediciones de la Presidencia de la República Bolivariana de Venezuela. Caracas, Venezuela.

3. Álvarez, M, (2008) Hacia un enfoque de la educación en competencias .Consejería de educación y ciencia del Principado de Asturias, España

4. Argudín, Y. (2014) Educación basada en Competencias Nociones y Antecedentes. Editorial TRILLAS. Distrito federal, México.

5. Aurelio, V, (2007) Aprendizaje Basado en competencias. Ediciones mensajero Universidad Deusto Bilbao.

6. Benito, A. y Cruz, A 2005. Las nuevas claves para la Docencia Universitaria. Editorial .Narcea, S, A. Madrid. España.

7. Bozo, Z (2009). El profesorado Universitario en la sociedad del conocimiento: competencias profesionales. Revista formación e innovación

8. Educativa Universitaria.Vol.2, Universidad Autónoma de Yucatán, Yucatán, México.

9. Cuello, Y Otros. 2002(www.ucv.ve/fileadmin/user_upload/.../Coello Sube y Ferrer). [Información en línea] [Consulta, julio 2017].

10. Chomsky, N. Aspects of Theory of syntax, MT Press, Cambrige, Mas, 1965.

11.Díaz -Barriga, F.y otros (1981). Metodología de diseño curricular para la educación superior. México. Trillas. 


\footnotetext{
SALUD Y VIDA. Revista Arbitrada Multidisciplinaria de Ciencias de la Salud

Volumen 3. Número 5. Año 3. Enero - Junio 2019. Santa Ana de Coro, Venezuela.

Hecho el depósito de Ley: FA2016000010

ISSN: 2610-8038

FUNDACIÓN KOINONIA (F.K). Santa Ana de Coro, Venezuela.

Reina Rivero Boyer
}

12. Fernández, A (2004). Universidad y currículo en Venezuela hacia el tercer milenio. Caracas: vicerrectorado académico/CEP-FHE.UCV. Caracas. Venezuela.

13.García, E (2010). Pedagogía constructivista y competencias . Editorial .Trillas. Ciudad de México, México.

14.González J y Wagenerar R 2003 Proyecto Tuning Educación Structur in Europa Universidad Deusto Foto composición IPAR S Coop. Bilbao. España.

15.Guedez, V. (1980). Lineamientos académicos para la definición de los perfiles académicos. Revista Curriculum.

16. Holdawa,E. First year at university; Perceptions and Experiences of students en Canadian journal of Higher Education,1987.

17.Yendrey, M (1999). Planning for competence, California State University and Sacramento, Prentice-Hall, Columbia, Ohio.

18. Martínez .M (1998) La investigación cualitativa Etnográfica .Editorial TRILLAS México

19. Morín, E (1999).Los Siete Saberes Necesarios para la Educación del futuro UNESCO adivirtual: unefm.edu.ve/moodle/medicina/file.php/1/medicina.html [17/04/17].

20.Mastorel, O. El profesor universitario sus competencias y formación .Revista curricular y formación vol. 15, diciembre 2011.

21.Palomares, A. (2007). Nuevos retos educativos, el modelo docente en el espacio europeo .Editorial Colecciones humanidades. 1era edición, España Madrid.

22.Parlamento Europeo y del Consejo (2006). Las competencias claves para el aprendizaje permanentehttp://eur-lex.europa.eu/legalcontent/ES/TXT/PDF/?uri=CELEX:32006H0962\&from=ES

23.Pírela, $F$ y otros (2006). Perfil de competencias del docente en la función de investigador y su relación con la producción intelectual consultado el www.serbi.luz.eduve/scielo php?script=sci_arttext\&pid=s1012 [información en línea] [17/04/17]. 


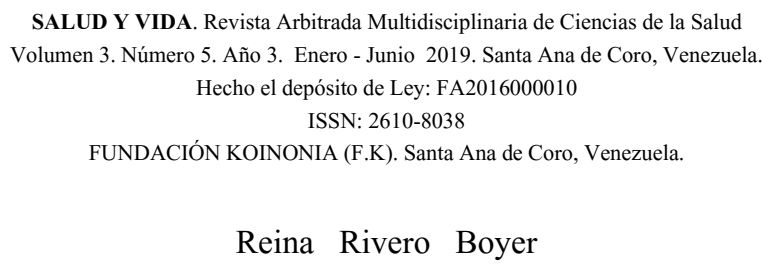

24. Quellet, A (2000), La evolución informática al servicio de las competencias, Revista escrita de administración de negocios N 41.pp 30-42.

25.Rivero, R (2015) .Diseño Instruccional de la unidad curricular Morfofisiopatologia Universidad Nacional Experimental Francisco de Miranda IX cohorte Prodinpa. Coro Venezuela

26.Sabino .El proceso de investigación .Editorial Panapo. Caracas Venezuela.

27.Tobón, S (2010) .Secuencia Didácticas: Aprendizaje y Evaluación de Competencias. ECO Ediciones. http://educacioncientifica.org/Refiedu/Vol2 2/REFIEDU 22 4.pdf consultado [10/05/17].

28. Tobón, S (2005).Formación Basada en competencias pensamiento complejo, diseño curricular y didáctica .Eco Ediciones. Bogotá. Colombia.

29. Unesco, (1998) Declaración Mundial sobre la Educación Superior en el siglo XXI Visión y acción, 9 de octubre de 1998.http://www.unesco.org/education/educprog/wche/declaration spa.htm [consulta: 13 mayo 17].

30. Unesco. (2009). La nueva dinámica de la educación superior y la investigación para el cambio social y el desarrollo. Conferencia mundial de educación superior .noviembre. Francia. [Consulta, 10 agosto 2017].

31.Zabalza, M (2007). Currículo Basado en competencias. Narceo Ediciones .Madrid. España.

(C2019 por el autor. Este artículo es de acceso abierto y distribuido según los términos y condiciones de la licencia Creative Commons Atribución-NoComercial-Compartirlgual 4.0 Internacional (CC BY-NC-

SA 4.0) (https://creativecommons.org/licenses/by-nc-sa/4.0/). 